\title{
Using differential transform method and Padé approximation for solving MHD three-dimensional Casson fluid flow past a porous linearly stretching sheet
}

\author{
Majeed A. Yousif ${ }^{a, *}$, Bewar A. Mahmood ${ }^{b}$, M. M. Rashidic,d \\ ${ }^{a}$ Department of Mathematics, Faculty of Science, University of Zakho, International Road Zakho-Duhok, P. O. Box 12, Duhok, Kurdistan \\ Region, Iraq. \\ ${ }^{b}$ Department of Mathematics, Faculty of Science, University of Duhok, Kurdistan Region, Iraq. \\ ' Shanghai Key Lab of Vehicle Aerodynamics and Vehicle Thermal Management Systems, Tongji University, 4800 Cao An Rd., Jiading, \\ Shanghai 201804, China. \\ ${ }^{d}$ ENN-Tongji Clean Energy Institute of Advanced Studies, Shanghai, China.
}

\begin{abstract}
The problem of MHD three-dimensional Casson fluid flow past a porous linearly stretching sheet is investigated analytically. Governing equations are reduced to a set of nonlinear ordinary differential equations using the similarity transformations, and solved via an efficient and suitable mathematical technique, named the differential transform method (DTM), in the form of convergent series, by applying Padé approximation. The results are compared with the results obtained by the shooting method of MATHEMATICA and with the fourth-order Runge-Kutta-Fehlberg results. The results of DTM-Padé are closer to numerical solutions than the results of DTM are. A comparison of our results with existing published results shows good agreement between them. Suitability end effectiveness of our method are illustrated graphically for various parameters. Moreover, it is also observed that the Casson fluid parameter, stretching parameter, Hartmann number and porosity parameter increase with increment in the velocity profiles. (C) 2017 All rights reserved.
\end{abstract}

Keywords: Casson model, three-dimensional flow, MHD flow, porous sheet, DTM- Padé. 2010 MSC: 35Q35, 80M25.

\section{Introduction}

In recent years non-Newtonian fluids have received more attention and signification than Newtonian fluids because of its various industrial, technological and natural applications. The flow of viscoelastic fluids was first presented by Casson in 1995. This model is cast off by fuel engineers in the description of adhesive slurry and is improved for forecasting high shear-rate viscosities when only low and transitional shear-rate data are accessible. The consideration of non-Newtonian fluid, in addition to classical Cauchy stress, has led to recent development of several theories [17, 20,30]. This model is cast off by fuel engineers

\footnotetext{
*Corresponding author

Email address: b.m.numerical@uod.ac (Majeed A. Yousif)
}

doi:10.22436/jmcs.017.01.15 
in the description of adhesive slurry and is improved for forecasting high shear-rate viscosities when only low and transitional shear-rate data are accessible [28]. Boundary layer flow over stretching plate was first presented by Crane [14]. Ece has studied the initial boundary layer flow past an impulsively started translating and spinning body of revolution [15]. Nazar et al. studied numerically, by Kellerbox method, the steady two-dimensional stagnation point flow of an incompressible micropolar fluid over a stretching sheet, when the sheet is stretched in its own plane [29]. Rashidi et al. investigated entropy generation in steady MHD flow, due to a rotating porous disk in a nanofluid [33]. Bhatti and Rashidi studied numerically MHD nanofluid towards a stagnation point flow, over a stretching surface [11]. Recently many authors discussed entropy generation of nanofluid (see $[1,4,9,10])$. Keimanesh et al. used the multi-step differential transform method to analyze the third grade non-Newtonian fluid flow between two parallel plates [25]. The effect of heat and mass transfer on peristaltic flow of particle fluid suspension with slip effects are examined in [12]. Also, nanofluid was investigated numerically for thermal radiation on MHD Carreau nanofluid towards a shrinking sheet and MHD stagnation-point flow over a permeable stretching/shrinking sheet in porous media with heat transfer [7, 8]. Makanda studied an effect of radiation on MHD free convection of a Casson fluid from a horizontal circular cylinder with partial slip in non-Darcy porous medium with viscous dissipation using BQLM numerical method [27]. Few recent studies related to non-Newtonian fluid are cited in $[18,19,24,26,37]$.

Most non-Newtonian fluids, Casson fluid and nanofluid are modeled by nonlinear differential equations. One of the famous and effective methods for solving nonlinear problems is the DTM, first proposed by Zhou [38], who solved some linear and nonlinear electrical-circuit problems. Chen and Ho developed this method for partial differential equations [13]. Ayaz applied it to a system of differential equations [3]. DTM has been applied to many problems such as linear partial differential equations of fractional order [31], nonlinear oscillatory systems [16], multi-order fractional differential equations [23], hyper-chaotic Rossler system [2], the fourth-order boundary value problems [22], and magnetohydrodynamics laminar viscous flow [32]. DTM constructs, for differential equations, an analytical solution in the form of power series. Furthermore, power series are not useful for large values of $\eta$, say $\eta \rightarrow \infty$. This can be attributed to the possibility that the radius of convergence may not be sufficiently large to contain the boundaries of the domain. Therefore, the combination of the series solution through the DTM or any other series solution method with the Padé approximation [5, 6] provides an effective tool for handling boundary-value problems on infinite or semi-infinite domains. DTM-Padé is a combination of DTM and Padé approximation. In recent years, the DTM-Padé has been successfully employed to solve many types of nonlinear problems such as MHD flow in a laminar liquid film [36], nano boundary-layers over stretching surfaces [34], heat transfer in a second-grade fluid through a porous medium [35] and off-centered stagnation flow toward a rotating disc [21].

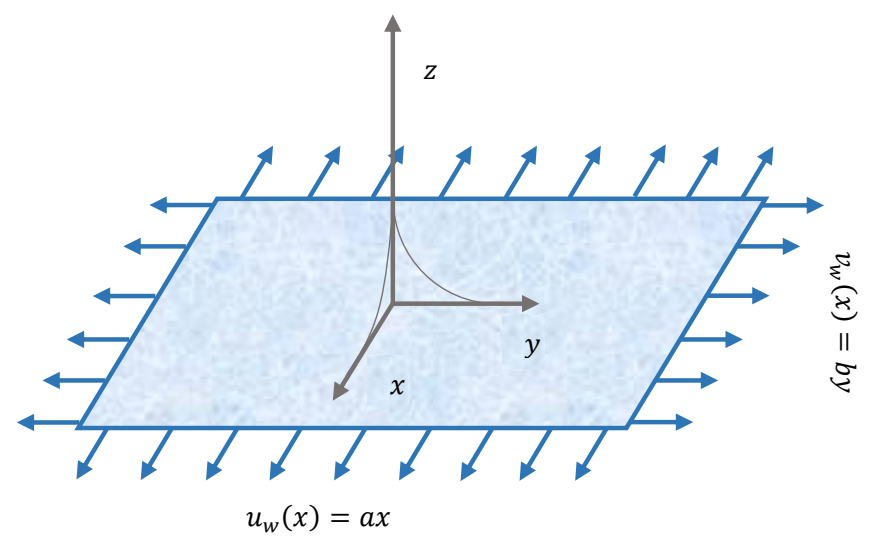

Figure 1: Nanofluid stretching sheet flow physical regime. 


\section{Nomenclature}

$\begin{array}{llll}x, y, \text { and } z & \text { direction of axes } & \tau_{w x} \text { and } \tau_{w y} & \text { wall shear stresses } \\ u, v, \text { and } w & \text { velocities along } x-, y-\text {, and } z \text { - axes } & f^{\prime}(\eta) \text { and } g^{\prime}(\eta) & \text { velocity profiles } \\ \mathrm{U}_{w} \text { and } \mathrm{V}_{w} & \text { stretching velocities } & \lambda & \text { porosity parameter } \\ v & \text { kinematic viscosity } & M & \text { Hartmann number } \\ \beta & \text { Casson fluid parameter } & c & \text { stretching parameter } \\ \mathrm{B}_{0} & \text { nanoparticle volume fraction } & \mathrm{a}, \mathrm{b} & \text { constants } \\ \mathrm{K} & \text { porous medium permeability } & & \end{array}$

The main purpose of the current study is to deliberate the three-dimensional examination for the Casson fluid model over the stretching sheet. We reduce the system of nonlinear partial differential equations to coupled nonlinear ordinary differential equations by diminishing. The Casson fluid parameter $b$, porosity parameter $k$, and Hartmann number $M$ are non-dimensionalized corporeal constraints. After transformation, nonlinear coupled equations are treated analytically to get series solutions using DTM and DTM-Padé, and a comparison with the available numerical methods in literature is presented. Also, each of the parameters is studied graphically.

\section{Mathematical model}

Consider a three-dimensional (3D) incompressible flow past a stretching sheet. It is assumed that the sheet stretches along the $x y$-plane, while the fluid is placed along the $z$-axis. Moreover, it is assumed that constant magnetic field applies normally to the fluid flow, and that the induced magnetic field is negligible. Here, we assume that the sheet stretches with the linear velocities $u=a x$ and $v=b y$ along the $x$ and $y$ axes, respectively (see Fig. 1). The boundary layer equations of three-dimensional incompressible Casson fluid are [28]

$$
\begin{aligned}
\frac{\partial u}{\partial x}+\frac{\partial v}{\partial y}+\frac{\partial w}{\partial z} & =0 \\
u \frac{\partial u}{\partial x}+v \frac{\partial u}{\partial y}+w \frac{\partial u}{\partial z} & =v\left(1+\frac{1}{\beta}\right)\left(\frac{\partial^{2} u}{\partial z^{2}}\right)-\frac{\sigma B^{2}}{\rho_{f}} u-\frac{v}{K} u, \\
u \frac{\partial v}{\partial x}+v \frac{\partial v}{\partial y}+w \frac{\partial v}{\partial z} & =v\left(1+\frac{1}{\beta}\right)\left(\frac{\partial^{2} v}{\partial z^{2}}\right)-\frac{\sigma B^{2}}{\rho_{f}} v-\frac{v}{K} v
\end{aligned}
$$

where $u, v$, and $w$ denote the velocities in the $x-, y-$, and $z$-directions, respectively, $\beta$ is the Casson fluid parameter, $v$ is kinematic viscosity, $B_{0}$ is the magnetic induction, and $K$ is the porous medium permeability. The associated boundary conditions of (2.1) and (2.2) are

$$
\begin{aligned}
& u=u_{w}(x)=a x, \quad v=v_{w}(x)=b x, \text { at } z=0, \\
& u \rightarrow 0, v \rightarrow 0, \text { at } z \rightarrow \infty .
\end{aligned}
$$

In the above expressions, $a$ and $b$ are positive constants, and $U_{w}$ and $v_{w}$ are stretching velocities in $x$ and $y$-directions, respectively. Introducing the following similarity transformations

$$
\begin{aligned}
& u=a x f^{\prime}(\eta), \quad v=b x g^{\prime}(\eta), \\
& w=-(a v)^{1 / 2}(f(\eta)+c g(\eta)), \\
& \eta=(a / v)^{1 / 2} z,
\end{aligned}
$$

where $c=b / a$ is the ratio of the velocities in $y$ - and $x$-directions, and prime denotes differentiation with respect to $\eta$. Making use of (2.4), the equation of continuity is identically satisfied, and (2.1) and (2.2) along with (2.3) take the following form

$$
\left(1+\frac{1}{\beta}\right) f^{\prime \prime \prime}-\left(f^{\prime}\right)^{2}+(f+g) f^{\prime \prime}-\left(M^{2}+\lambda\right) f^{\prime}=0,
$$




$$
\begin{aligned}
& \left(1+\frac{1}{\beta}\right) g^{\prime \prime \prime}-\left(g^{\prime}\right)^{2}+(f+g) g^{\prime \prime}-\left(M^{2}+\lambda\right) g^{\prime}=0, \\
& f(\eta)=0, f^{\prime}(\eta)=1, \quad g(\eta)=0 \quad g^{\prime}(\eta)=c, \quad \text { at } \eta=0, \\
& f^{\prime}(\eta)=0, \quad g^{\prime}(\eta)=0, \text { at } \eta \rightarrow \infty .
\end{aligned}
$$

In these expressions, $M^{2}=\frac{\sigma B_{0}^{2}}{\rho a}$ is the magnetic parameter, and $\lambda=\frac{\alpha}{a K}$ is the porosity parameter.

Expressions for the skin friction coefficient $C_{f}$ on the surface along the $x$ - and $y$-directions, which are denoted by $C_{f x}$ and $C_{f y}$, respectively, are defined by

$$
C_{f x}=\frac{\tau_{w x}}{\rho u_{w}^{2}}, \quad C_{f y}=\frac{\tau_{w y}}{\rho u_{w}^{2}},
$$

where $\tau_{w x}$ and $\tau_{w y}$ are the wall shear stresses along $x$ and $y$-direction, respectively. Using the variables (2.4), we obtain

$$
\operatorname{Re}_{x}^{1 / 2} C_{f x}=\left(1+\frac{1}{\beta}\right) f^{\prime \prime \prime}(0), \quad \operatorname{Re}_{x}^{1 / 2} C_{f y}=\left(1+\frac{1}{\beta}\right)\left(c \frac{y}{x}\right) g^{\prime \prime}(0),
$$

where $R_{e x}=u_{x}(x) x / v$ is local Reynolds number based on the stretching velocity $u_{w}(x)$.

\section{Analytical approximations by means of the DTM-Padé}

Taking the one-dimensional differential transform, from Table 1 to each term of (2.5)-(2.6), the following transforms are obtained

Table 1: The operations for the one-dimensional DTM.

\begin{tabular}{ll}
\hline Original function & Transformed function \\
\hline$w(x)=u(x) \pm v(x)$ & $W(k)=U(k) \pm V(k)$ \\
$w(x)=\lambda u(x)$ & $W(k)=\lambda u(k), \lambda$ is a constant \\
$w(x)=\frac{d u(x)}{d x}$ & $W(k)=(k+1) u(k+1)$ \\
$w(x)=\frac{d^{r} u(x)}{d x^{r}}$ & $W(k)=(k+1)(k+2) \ldots(k+r) u(k+r)$ \\
$w(x)=u(x) v(x)$ & $W(k)=\sum_{r=0}^{r} u(r) v(k-r)$ \\
$w(x)=\frac{d u(x)}{d x} \frac{d v(x)}{d x}$ & $W(k)=\sum_{r=0}^{r}(r+1)(k-r+1) u(r+1) V(k-r+1)$ \\
$w(x)=u(x) \frac{d v(x)}{d x}$ & $W(k)=\sum_{r=0}^{k}(k-r+1) u(r) V(k-r+1)$ \\
$w(x)=u(x) \frac{d^{2} u(x)}{d x^{2}}$ & $W(k)=\sum_{r=0}^{k}(k-r+2)(k-r+1) u(r) V(k-r+2)$ \\
\hline
\end{tabular}

$$
\begin{aligned}
f^{\prime \prime \prime} & \rightarrow(k+1)(k+2)(k+3) F(k+3), \\
f^{\prime \prime} & \rightarrow \sum_{r=0}^{k}(k-r+1)(k-r+2) F(r) F(k-r+2), \\
f^{\prime 2} & \rightarrow \sum_{r=0}^{k}(r+1) F(r+1)(k-r+1) F(k-r+1), \\
g^{\prime \prime \prime} & \rightarrow(k+1)(k+2)(k+3) G(k+3), \\
g g^{\prime \prime} & \rightarrow \sum_{r=0}^{k}(k-r+1)(k-r+2) G(r) G(k-r+2), \\
g^{\prime 2} & \rightarrow \sum_{r=0}^{k}(r+1) G(r+1)(k-r+1) G(k-r+1),
\end{aligned}
$$




$$
\begin{aligned}
& g f^{\prime \prime} \rightarrow \sum_{r=0}^{k}(k-r+1)(k-r+2) G(r) F(k-r+2), \\
& f g^{\prime \prime} \rightarrow \sum_{r=0}^{k}(k-r+1)(k-r+2) F(r) G(k-r+2),
\end{aligned}
$$

where $F(k)$ and $G(k)$ are the transformed functions of $f(k)$ and $g(k)$, respectively and are given by

$$
\begin{aligned}
& f(\eta)=\sum_{k=0}^{\infty} F(k) \eta^{k}, \\
& g(\eta)=\sum_{k=0}^{\infty} G(k) \eta^{k} .
\end{aligned}
$$

Substituting (3.1) and (3.2) into (2.5) and (2.6) and using boundary conditions of (2.7) and (2.8) we get

$$
\begin{aligned}
&\left(\frac{1}{\beta}+1\right)(k+3)(k+2)(k+1) F(k+3) \\
&= \sum_{r=0}^{k}(r+1) F(r+1)(k-r+1) F(k-r+1) \\
&-\sum_{r=0}^{k} F(r)(k-r+2)(k-r+1) F(k-r+2) \\
&-\sum_{r=0}^{k} G(r)(k-r+2)(k-r+1) F(k-r+2)+\left(\lambda+M^{2}\right)(k+1) F(k+1), \\
&\left(\frac{1}{\beta}+1\right)(k+3)(k+2)(k+1) G(k+3) \\
&= \sum_{r=0}^{k}(r+1) G(r+1)(k-r+1) G(k-r+1) \\
&-\sum_{r=0}^{k} F(r)(k-r+2)(k-r+1) G(k-r+2) \\
&-\sum_{r=0}^{k} G(r)(k-r+2)(k-r+1) G(k-r+2)+\left(\lambda+M^{2}\right)(k+1) G(k+1), \\
& F(0)=0, \quad F(1)=1, \quad F(2)=a, \\
& G(0)=0, \quad G(1)=0.5, \quad G(2)=b .
\end{aligned}
$$

Moreover, substituting (3.7) and (3.8) into (3.5) and (3.6), by a recursive method we can calculate the values of $F(k)$ and $G(k)$.

Hence, substituting all $F(k)$ and $G(k)$ into (3.3) and (3.4), we get the series solutions

$$
\begin{aligned}
& f(\eta) \cong+\eta+a \eta^{2}+\frac{\left(1+\lambda+M^{2}\right)}{6\left(1+\frac{1}{\beta}\right)} \eta^{3}+\frac{\left(2 a\left(\lambda+M^{2}\right)+a\right)}{24\left(1+\frac{1}{\beta}\right)} \eta^{4}+\cdots, \\
& g(\eta) \cong 0.5 \eta+b \eta^{2}+\frac{\left(0.5\left(0.25+\lambda+M^{2}\right)\right)}{6\left(1+\frac{1}{\beta}\right)} \eta^{3}+\frac{\left(2 b\left(\lambda+M^{2}\right)-b\right)}{24\left(1+\frac{1}{\beta}\right)} \eta^{4}+\cdots .
\end{aligned}
$$

The best way to enlarge the convergence radius of the truncated series solution is the Pade approximation where the polynomial approximant converts into a ratio of two polynomials. Without using the Padé approximation, the analytical solution obtained by the DTM, cannot satisfy boundary conditions at infinity. It is therefore essential to combine the series solution, obtained by the DTM with the Padé approximation 
to provide an effective tool to handle boundary value problems in infinite domains. Hence applying the Padé approximation to (3.9) and (3.10) and using asymptotic boundary conditions (2.7) and (2.8) at $\eta=\infty$, we can obtain $a$ and $b$.

For example, the values of $a=-0.491568$ and $b=-0.223689$, are obtained after applying DTM-Padé when $M=\lambda=c=0.5$ and $\beta=1$.

\section{Results and discussion}

In the present section, we discuss the velocity profiles $f^{\prime}(\eta)$ and $g^{\prime}(\eta)$ for various physical parameters such as the Casson fluid parameter $\beta$, Hartmann number $M$, porosity parameter $\lambda$, and stretching ratio $c$. In order to verify the accuracy of the present method, we compare some of our results with the numerical results obtained by the shooting Runge-Kutta technique in MATHEMATICA. Table 2, Fig. 2, and Fig. 3 display a comparison for non-dimensional velocity profiles $f^{\prime}(\eta)$ and $g^{\prime}(\eta)$ between the DTM, DTM-Padé, and numerical solution results based on the shooting technique obtained by MATHEMATICA, and it can be seen that there is an excellent agreement between DTM-Padé and numerical solution, but when $\eta \geqslant 2.9$ DTM solution will be far from numerical solution. Fig. 4 and Fig. 5 show the effects of nonNewtonian parameter $\beta$ on the velocity profiles $f^{\prime}(\eta)$ and $g^{\prime}(\eta)$. It can be seen that when we increase the non-Newtonian parameter $\beta$ indefinitely, the present phenomena obviously reduce to Newtonian fluid. Also, it is clear that increasing of non-Newtonian parameter $\beta$ produces resistance in the fluid flow. From Table 3 it can be seen that an excellent correlation has been achieved in the recent work of Nadeem et al. [28] for $-\left(1+\frac{1}{\beta}\right) f^{\prime \prime}(0)$ and $-\left(1+\frac{1}{\beta}\right) g^{\prime \prime}(0)$, when $M=0, c=0.5$, and $\beta=1$. From Fig. 6 and Fig. 7 , it is clear that the velocities in both directions decrease when increasing the values of the porosity parameter $\lambda$, within the boundary layer. Also for higher values of $\lambda$, the thickness boundary layer decreases. From Fig. 8 and Fig. 9 it is clear that both boundary layer thickness and the magnitude of the velocity reduce when the values of $M$ increase. Fig. 10 and Fig. 11 show that the velocity $f^{\prime}(\eta)$ reduce at increasing the values of the stretching parameter $c$, while $g^{\prime}(\eta)$ varies with respect to various values of the stretching parameter $\mathrm{c}$.

Table 2: Comparison between the results of DTM-Padé $[15,15]$ and the numerical shooting method obtained by MATHEMATICA, when $\lambda=M=c=0.5$, and $\beta=1$.

\begin{tabular}{l|ll|ll}
\hline \hline & \multicolumn{2}{|c|}{$f^{\prime}(\eta)$} & \multicolumn{2}{c}{$g^{\prime}(\eta)$} \\
\hline$\eta$ & DTM-Padé[15,15] & Numerical & DTM-Padé[15,15] & Numerical \\
\hline 0.0 & 1.000000 & 1.000000 & 0.500000 & 0.500000 \\
0.5 & 0.605502 & 0.605502 & 0.313537 & 0.313537 \\
1.0 & 0.360965 & 0.360965 & 0.190919 & 0.190917 \\
1.5 & 0.213035 & 0.213035 & 0.114108 & 0.114106 \\
2.0 & 0.124921 & 0.124921 & 0.067412 & 0.067409 \\
2.5 & 0.072927 & 0.072927 & 0.039527 & 0.039523 \\
3.0 & 0.042410 & 0.042409 & 0.023047 & 0.023042 \\
3.5 & 0.024544 & 0.024543 & 0.013360 & 0.013354 \\
4.0 & 0.014088 & 0.014088 & 0.007679 & 0.007671 \\
\hline \hline
\end{tabular}

Table 3: Comparison between the results of DTM-Padé[15,15] and the numerical solution obtained by the fourth-order RungeKutta-Fehlberg (see [28]) for the skin friction coefficients, when $M=0, c=0.5$, and $\beta=1$.

\begin{tabular}{l|ll|ll}
\hline \hline & Numerical & present work & Numerical & present work \\
\hline$\lambda$ & $-\left(1+\frac{1}{\beta}\right) f^{\prime \prime}(0)$ & $-\left(1+\frac{1}{\beta}\right) f^{\prime \prime}(0)$ & $-\left(1+\frac{1}{\beta}\right) g^{\prime \prime}(0)$ & $-\left(1+\frac{1}{\beta}\right) g^{\prime \prime}(0)$ \\
\hline 0 & 1.5459 & 1.5472 & 0.6579 & 0.6589 \\
0.5 & 1.8361 & 1.83633 & 0.8228 & 0.8230 \\
1 & 2.0884 & 2.0885 & 0.9614 & 0.9614 \\
\hline \hline
\end{tabular}




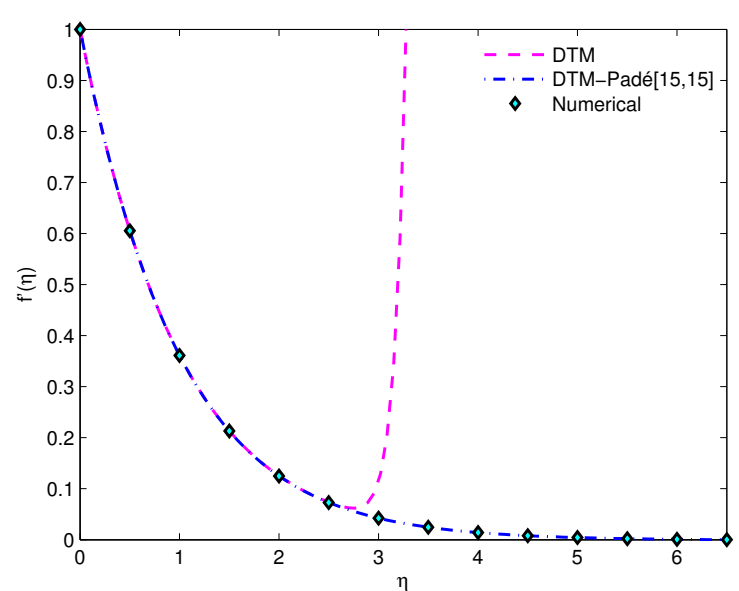

Figure 2: Velocity $f^{\prime}(\eta)$ obtained by the DTM and the DTMPadé[15,15] and comparison with the numerical solution, when $\lambda=M=c=0.5$, and $\beta=1$.

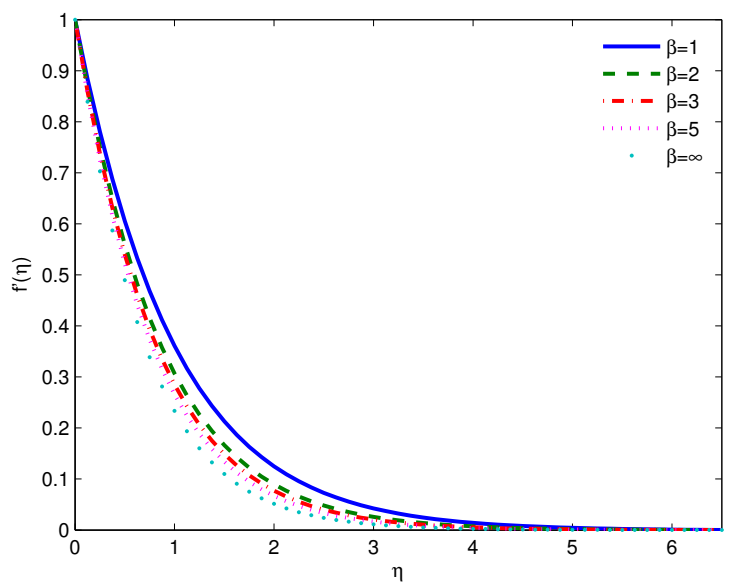

Figure 4: Effect of Casson fluid parameter $(\beta)$ on velocity $f^{\prime}(\eta)$, when $\lambda=M=c=0.5$.

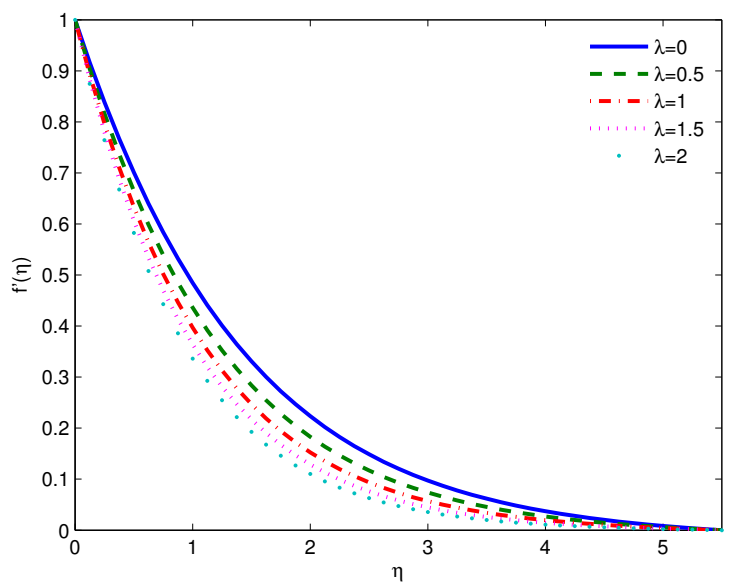

Figure 6: Effect of porosity parameter $(\lambda)$ on velocity $f^{\prime}(\eta)$, when $M=c=0.5$ and $\beta=1$.

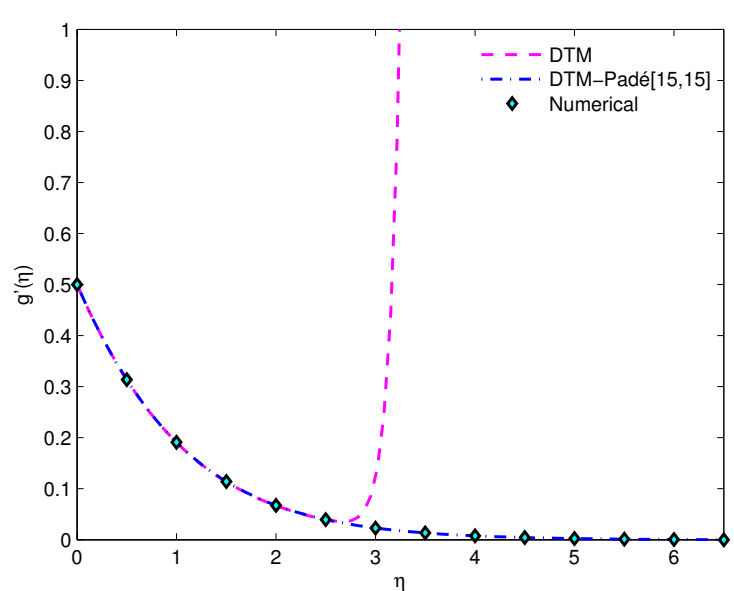

Figure 3: Velocity $g^{\prime}(\eta)$ obtained by the DTM and the DTMPadé[15,15] and comparison with the numerical solution, when $\lambda=M=c=0.5$, and $\beta=1$.

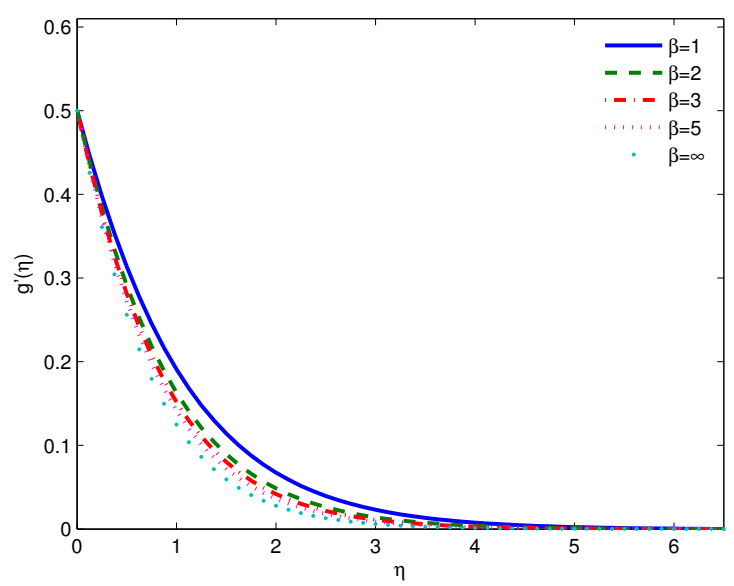

Figure 5: Effect of Casson fluid parameter $(\beta)$ on velocity $g^{\prime}(\eta)$, when $\lambda=M=c=0.5$.

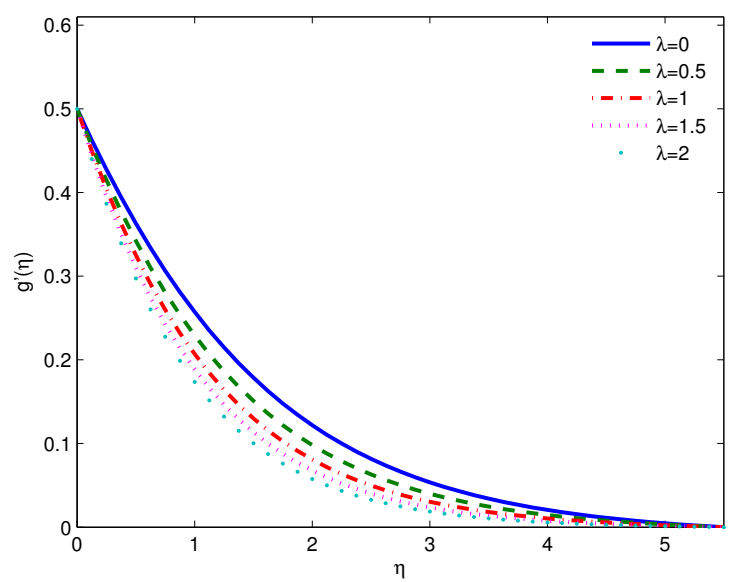

Figure 7: Effect of porosity parameter $(\lambda)$ on velocity $g^{\prime}(\eta)$, when $M=c=0.5$ and $\beta=1$. 


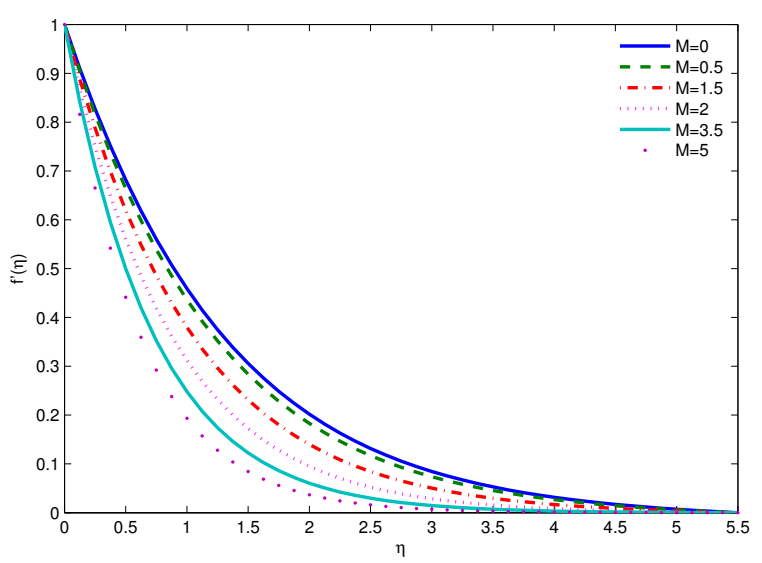

Figure 8: Effect of Hartmann number $(M)$ on velocity $f^{\prime}(\eta)$, when $\lambda=\mathrm{c}=0.5$ and $\beta=1$.

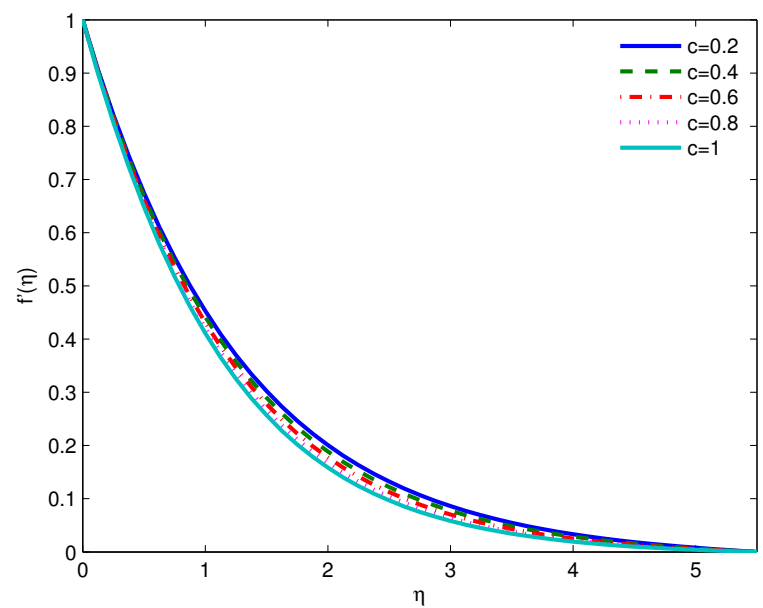

Figure 10: Effect of stretching parameter $(c)$ on velocity $f^{\prime}(\eta)$, when $\lambda=M=0.5$ and $\beta=1$.

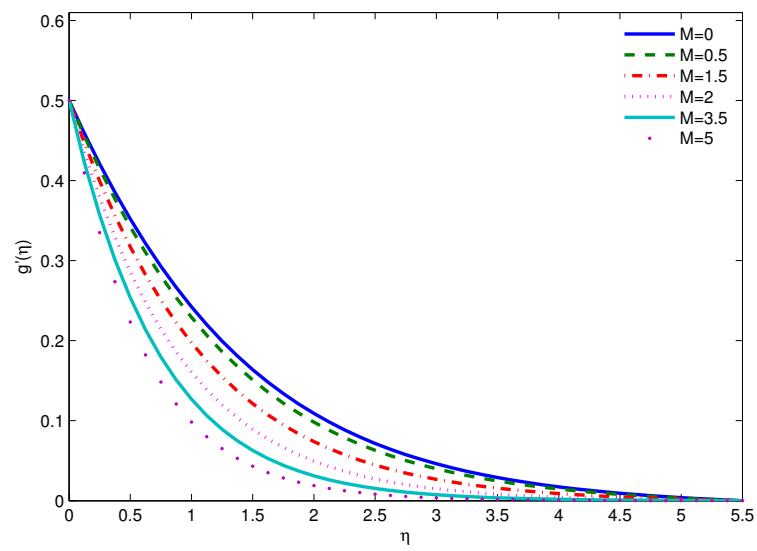

Figure 9: Effect of Hartmann number $(M)$ on velocity $g^{\prime}(\eta)$, when $\lambda=\mathrm{c}=0.5$ and $\beta=1$.

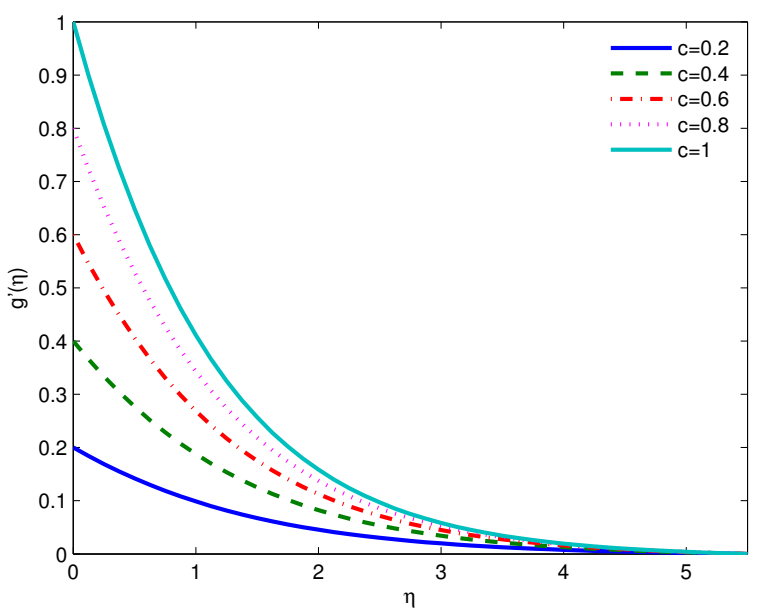

Figure 11: Effect of stretching parameter $(c)$ on velocity $g^{\prime}(\eta)$, when $\lambda=M=0.5$ and $\beta=1$.

\section{Conclusion}

We solved analytically MHD boundary layer three-dimensional flow for Casson fluid model over a stretching sheet by a new and effective method called DTM-Padé. It was found that DTM-Padé is a powerful method for solving problems consisting of systems of nonlinear differential equations. The method was applied directly without requiring linearization, discretization, or perturbation. The main results are:

- The DTM-Padé results have very good agreement with the shooting method results obtained by MATHEMATICA and the fourth-order Runge-Kutta-Fehlberg results.

- Various values of parameters $\beta, \lambda, M$, and $c$ affect the velocities $f^{\prime}(\eta)$ and $g^{\prime}(\eta)$.

- Without using the Padé approximation, the analytical solution obtained by the DTM can not satisfy boundary conditions at infinity.

- The results of DTM-Padé are closer to numerical solutions then the results of DTM are. 


\section{References}

[1] T. Abbas, M. Ayub, M. M. Bhatti, M. M. Rashidi, M. E. S. Ali, Entropy generation on nanofluid flow through a horizontal riga plate, Entropy, 18 (2016), 223. 1

[2] M. M. Al-Sawalha, M. S. M. Noorani, Application of the differential transformation method for the solution of the hyperchaotic Rössler system, Commun. Nonlinear Sci. Numer. Simul., 14 (2009), 1509-1514. 1

[3] F. Ayaz, Solutions of the system of differential equations by differential transform method, Appl. Math. Comput., 147 (2004), 547-567. 1

[4] M. Ayub, T. Abbas, M. M. Bhatti, Inspiration of slip effects on electromagnetohydrodynamics (EMHD) nanofluid flow through a horizontal Riga plate, Eur. Phys. J. Plus, 131 (2016), 1-9. 1

[5] G. A. Baker, Essentials of Padé approximants, Academic Press, London, (1975). 1

[6] G. A. Baker, P. Graves-Morris, Padé approximants, Parts I and II, Encycl. Math. Appl., 13 and 14, Reading, Mass., Addison-Wesley, New York, (1981). 1

[7] M. M. Bhatti, T. Abbas, M. M. Rashidi, A new numerical simulation of MHD stagnation-point flow over a permeable stretching/shrinking sheet in porous media with heat transfer, Iran. J. Sci. Technol. Trans. A Sci., (2016), 1-7. 1

[8] M. M. Bhatti, T. Abbas, M. M. Rashidi, M. E. S. Ali, Numerical simulation of entropy generation with thermal radiation on MHD Carreau nanofluid towards a shrinking sheet, Entropy, 18 (2016), 200. 1

[9] M. M. Bhatti, T. Abbas, M. M. Rashidi, M. E. S. Ali, Z. G. Yang, Entropy generation on MHD Eyring-Powell nanofluid through a permeable stretching surface, Entropy, 18 (2016), 224. 1

[10] M. M. Bhatti, M. M. Rashidi, Entropy generation with nonlinear thermal radiation in MHD boundary layer flow over a permeable shrinking/stretching sheet: numerical solution, J. Nanofluids, 5 (2016), 543-548. 1

[11] M. M. Bhatti, M. M. Rashidi, Numerical simulation of entropy generation on MHD nanofluid towards a stagnation point flow over a stretching surface, Int. J. Appl. Comput. Math., 2 (2016), 1-15. 1

[12] M. M. Bhatti, A. Zeeshan, Heat and mass transfer analysis on peristaltic flow of particle-fluid suspension with slip effects, J. Mech. Med. Biol., 17 (2016), 16 pages. 1

[13] C. K. Chen, S. H. Ho, Solving partial differential equations by two-dimensional differential transform method, Appl. Math. Comput., 106 (1999), 171-179. 1

[14] L. Crane, Flow past a stretching plate, Z. Angew. Math. Phys., 21 (1970), 645-647. 1

[15] M. C. Ece, The initial boundary-layer flow past a translating and spinning rotational symmetric body, J. Engrg. Math., 26 (1992), 415-428. 1

[16] M. El-Shahed, Application of differential transform method to non-linear oscillatory systems, Commun. Nonlinear Sci. Numer. Simul., 13 (2008), 1714-1720. 1

[17] R. Ellahi, Effects of the slip boundary condition on non-Newtonian flows in a channel, Commun. Nonlinear Sci. Numer. Simul., 14 (2009), 1377-1384. 1

[18] R. Ellahi, The effects of MHD and temperature dependent viscosity on the flow of non-Newtonian nanofluid in a pipe: analytical solutions, Appl. Math. Model., 37 (2013), 1451-1467. 1

[19] R. Ellahi, S. Aziz, A. Zeeshan, Non-Newtonian nanofluid flow through a porous medium between two coaxial cylinders with heat transfer and variable viscosity, J. Porous Media, 16 (2013), 205-216. 1

[20] R. Ellahi, A. Riaz, Analytical solutions for MHD flow in a third-grade fluid with variable viscosity, Math. Comput. Modelling, 52 (2010), 1783-1793. 1

[21] E. Erfani, M. M. Rashidi, A. B. Parsa, The modified differential transform method for solving off-centered stagnation flow toward a rotating disc, Int. J. Comput. Methods, 7 (2010), 655-670. 1

[22] V. S. Ertürk, S. Momani, Comparing numerical methods for solving fourth-order boundary value problems, Appl. Math. Comput., 188 (2008), 1963-1968. 1

[23] V. S. Ertürk, S. Momani, Z. Odibat, Application of generalized differential transform method to multi-order fractional differential equations, Commun. Nonlinear Sci. Numer. Simul., 13 (2008), 1642-1654. 1

[24] P. K. Kameswaran, S. Shaw, P. Sibanda, P. V. S. N. Murthy, Homogeneous-heterogeneous reactions in a nanofluid flow due to a porous stretching sheet, Int. J. Heat Mass Transf., 57 (2013), 465-472. 1

[25] M. Keimanesh, M. M. Rashidi, A. J. Chamkha, R. Jafari, Study of a third grade non-Newtonian fluid flow between two parallel plates using the multi-step differential transform method, Comput. Math. Appl., 62 (2011), 2871-2891 1

[26] A. A. Khan, R. Ellahi, M. Usman, The effects of variable viscosity on the peristaltic flow of non-Newtonian fluid through a porous medium in an inclined channel with slip boundary conditions, J. Porous Media, 16 (2013), 59-67. 1

[27] G. Makanda, S. Shaw, P. Sibanda, Effects of radiation on MHD free convection of a Casson fluid from a horizontal circular cylinder with partial slip in non-Darcy porous medium with viscous dissipation, Bound. Value Probl., 2015 (2015), 14 pages. 1

[28] S. Nadeem, R. U. Haq, N. S. Akbar, Z. H. Khan, MHD three-dimensional Casson fluid flow past a porous linearly stretching sheet, Alexandria Eng. J., 52 (2013), 577-582. 1, 2, 4, 3

[29] R. Nazar, N. Amin, D. Filip, I. Pop, Stagnation point flow of a micropolar fluid towards a stretching sheet, Int. J. Nonlinear Mech., 39 (2004), 1227-1235. 1

[30] M. V. Ochoa, Analysis of drilling fluid rheology and tool joint effect to reduce errors in hydraulics calculations, Ph.D. Diss., Texas A\&M University, (2006). 1 
[31] Z. Odibat, S. Momani, A generalized differential transform method for linear partial differential equations of fractional order, Appl. Math. Lett., 21 (2008), 194-199. 1

[32] A. B. Parsa, M. M. Rashidi, O. A. Bég, S. M. Sadri, Semi-computational simulation of magneto-hemodynamic flow in a semi-porous channel using optimal homotopy and differential transform methods, Comput. Biol. Med., 43 (2013), 1142-1153. 1

[33] M. M. Rashidi, S. Abelman, N. Freidooni Mehr, Entropy generation in steady MHD flow due to a rotating porous disk in a nanofluid, Int. J. Heat Mass Transf., 62 (2013), 515-525. 1

[34] M. M. Rashidi, E. Erfani, The modified differential transform method for investigating nano boundary-layers over stretching surfaces, Internat. J. Numer. Methods Heat Fluid Flow, 21 (2011), 864-883. 1

[35] M. M. Rashidi, T. Hayat, T. Keimanesh, H. Yousefian, A study on heat transfer in a second-grade fluid through a porous medium with the modified differential transform method, Heat Trans. Asian Res., 42 (2013), 31-45. 1

[36] M. M. Rashidi, M. Keimanesh, Using differential transform method and Padé approximant for solving mhd flow in a laminar liquid film from a horizontal stretching surface, Math. Probl. Eng., 2010 (2010), 14 pages. 1

[37] S. Shaw, P. K. Kameswaran, P. Sibanda, Homogeneous-heterogeneous reactions in micropolar fluid flow from a permeable stretching or shrinking sheet in a porous medium, Bound. Value Probl., 2013 (2013), 10 pages. 1

[38] J. K. Zhou, Differential transformation and its applications for electrical circuits, Huazhong University Press, Wuhan, China, (1986) (in Chinese). 1 